\title{
Multipolar pacing by cardiac resynchronization therapy with a defibrillators treatment in type 2 diabetes mellitus failing heart patients: impact on responders rate, and clinical outcomes
}

\author{
Celestino Sardu ${ }^{1,2,3^{*}}$, Michelangela Barbieri ${ }^{1}$, Matteo Santamaria², Valerio Giordano ${ }^{2}$, Cosimo Sacra ${ }^{2}$, \\ Pasquale Paolisso ${ }^{1}$, Alessandro Spirito ${ }^{3}$, Raffaele Marfella', Giuseppe Paolisso ${ }^{1}$ and Maria Rosaria Rizzo ${ }^{1}$
}

\begin{abstract}
Background: Type 2 diabetes mellitus (T2DM) is a multi factorial disease, affecting clinical outcomes in failing heart patients treated by cardiac resynchronization therapy with a defibrillator (CRT-d).

Methods: One hundred and ninety-five T2DM patients received a CRT-d treatment. Randomly the study population received a CRT-d via multipolar left ventricle (LV) lead pacing (n 99, multipolar group), vs a CRT-d via bipolar LV pacing ( $n$ 96, bipolar group). These patients were followed by clinical, and instrumental assessment, and telemetric device control at follow up. In this study we evaluated, in a population of failing heart T2DM patients, cardiac deaths, all cause deaths, arrhythmic events, CRT-d responders rate, hospitalizations for HF worsening, phrenic nerve stimulation (PNS), and LV catheter dislodgment events (and re-intervention for LV catheter re-positioning), comparing multipolar CRT-d vs bipolar CRT-d group of patients at follow up.
\end{abstract}

Results: At follow up there was a statistical significant difference about atrial arrhythmic events [7 (7\%) vs 16 (16.7\%), p value 0.019], hospitalizations for HF worsening [15 (15.2\% vs 24 (25\%), p value 0.046], LV catheter dislodgments [1 (1\%) vs 9 (9.4\%), p value 0018], PNS [5 (5\%) vs 18 (18.7\%), p value 0.007], and LV re-positioning [1 (1\%) vs 9 (9.4\%), p value 0.018], comparing multipolar CRT-d vs bipolar CRT-d group of patients. Multipolar pacing was an independent predictor of all these events.

Conclusions: CRT-d pacing via multipolar LV lead vs bipolar LV lead may reduce arrhythmic burden, hospitalization rate, PNS, LV catheters dislodgments, and re-interventions in T2DM failing heart patients.

Clinical trial number NCT03095196

\section{Background}

Type 2 diabetes mellitus (T2DM) is a worldwide increasing disease [1]. T2DM may impact on heart functions [2], leading to cardiovascular disease [3]. An evident

\footnotetext{
*Correspondence: drsarducele@gmail.com

${ }^{1}$ Department of Medical, Surgical, Neurological, Metabolic and Aging Sciences, University of Campania "Luigi Vanvitelli", Piazza Miraglia, 2, 80138 Naples, Italy

Full list of author information is available at the end of the article
}

correlation exists between T2DM, and heart failure (HF) disease [4]. In fact, T2DM may condition HF disease progression, its clinical stage, and the response to therapeutic treatments [4]. In HF patients, cardiac resynchronization therapy with a defibrillator (CRT-d) is a well-established treatment to improve symptoms, quality of life, New York Heart Association (NYHA) class, and clinical outcomes [5]. On other hand, the T2DM clinical stage, the glucose homeostasis, the insulin therapy, and the ageing may affect CRT-d response, and the related 
clinical outcomes [6-12]. In last decades, the advancement of CRT-d technology worked to reduce implant complications, as phrenic nerve stimulation (PNS), and left ventricle (LV) leads dislodgments [13], and to improve clinical outcomes in CRT-d patients [14]. In this setting, CRT-d pacing via a multipolar LV lead may represent one of these technological advancements. In fact, the stable and continuous CRT- $d$ pacing by multipolar LV lead looked to condition the clinical prognosis, and CRT-d responders rate in failing heart patients [14]. At our knowledge, there are not studies investigating these effects in a population of T2DM failing heart subjects. Moreover, our study hypothesis was that, in T2DM failing heart patients multipolar LV pacing may lead to a reduction of PNS episodes, LV leads dislodgments, and interventions to re-positioning LV leads as compared to bipolar LV pacing. These beneficial effects may be associated to a significant reduction of arrhythmic events, hospitalizations for HF worsening, cardiac deaths, and all cause deaths in T2DM patients. Therefore, the aim of the study was to investigate these effects in a population of T2DM failing heart patients randomly treated by CRT-d via multipolar LV lead vs bipolar LV lead. In T2DM failing heart patients, CRT-d via multipolar LV lead vs bipolar LV lead may induce an amelioration of the HF clinical status, and of the CRT-d responders rate.

\section{Methods}

From September 2012 to September 2015 we conducted a multicenter, prospective, randomized study at University of Campania Luigi Vanvitelli, Italy, Catholic University of Sacred Heart, Campobasso, Italy, and John Paul II Research and Care Foundation, Campobasso, Italy. We screened 213 consecutive T2DM patients (screening phase) with stable chronic heart failure, New York Heart Association (NYHA) functional class II or III, left bundle branch block, severe left ventricle ejection fraction reduction (LVEF $<35 \%$ ), stable sinus rhythm, candidates to receive a CRT-d treatment according to the international guidelines [15, 16] (Fig. 1). Exclusion criteria were as follows: age $<18$ or $>75$ years, ejection fraction $>35 \%$, previous implantable cardioverter defibrillator (ICD), CRT-d and/or pacemaker implant, absence of informed patient consent, and any condition that would make survival for 1 year unlikely. One hundred and ninety-nine eligible patients were included in the study, and received a CRT-d treatment, and a traditional CRT-d ambulatory monitoring (inclusion phase) (Fig. 1). The CRT-d has randomly undergone via multipolar LV lead pacing ( $\mathrm{n} \mathrm{101)}$ vs bipolar LV lead pacing (n 98) (intervention phase) (Fig. 1). We used a computer programming code for treatment randomization. Study population was then divided in multipolar CRT-d group (multipolar LV

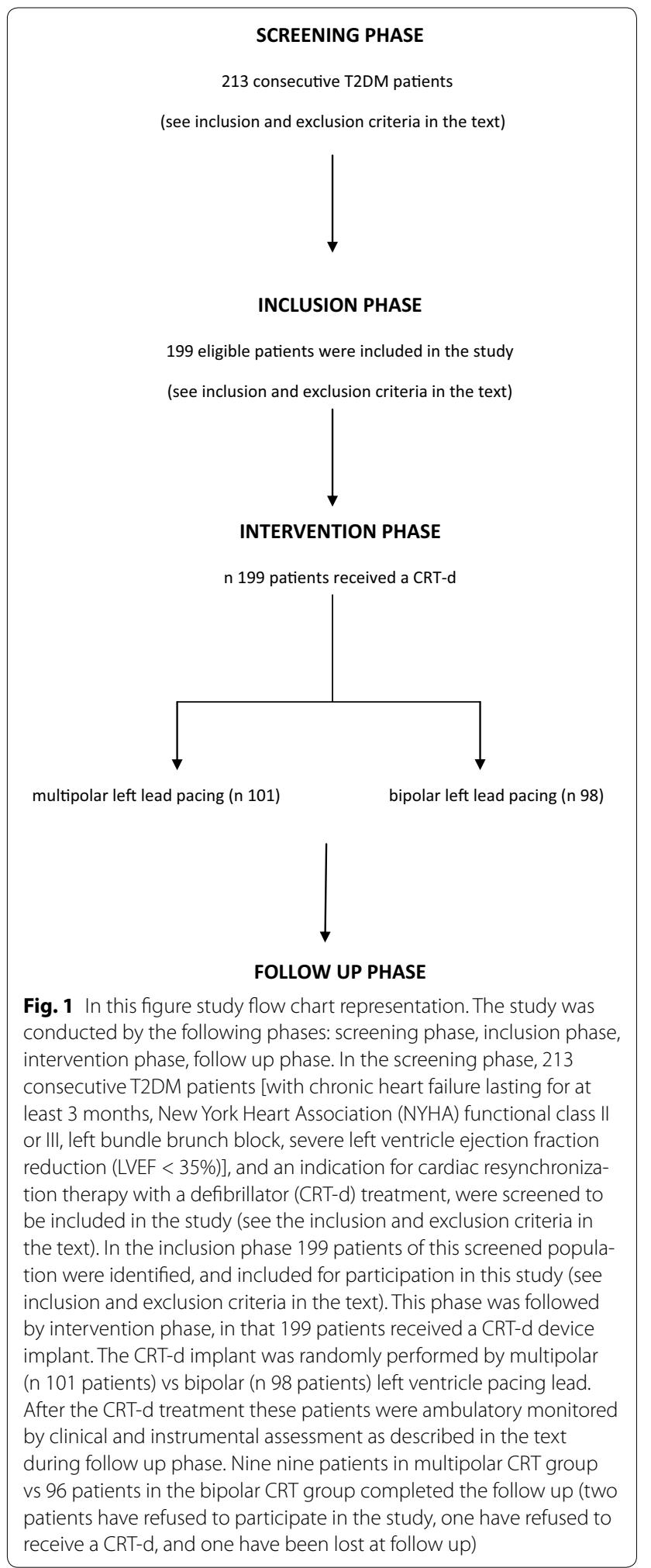

lead pacing), vs bipolar CRT-d group (bipolar LV lead pacing). Before interventions, baseline laboratory studies, including HbA1c, lipid panel, and fibrinogen, were 
determined. In this population two patients have refused to participate in the study, one have refused to receive a CRT-d, and one have been lost at follow up. Therefore, 99 patients in multipolar CRT group vs 96 patients in the bipolar CRT group completed the 12 months follow up (follow up phase) (Fig. 1). Responders patients to a CRT-d treatment were defined by evidence of LV reverse remodeling, 6 min-walk improvement and Minnesota living with heart failure scale improvement as previous described [15, 16]. Enrolled patients were followed by clinical, instrumental assessment, and device telemetric control (at implant, 10 days, 6th, and 12th months after discharge). During these visits, and device interrogations, we reported lead functionality parameters, and arrhythmic events in CRT-d recipients, PNS episodes, and subsequently CRT-d effect in terms of clinical outcomes, as CRT response entity, and clinical events. The study was conducted in accordance with the Declaration of Helsinki. The Ethics Committees of all participating institutions approved the protocol. All patients were informed about the study nature, and gave their written informed, and signed consent to participate in the study.

\section{Intervention phase CRT-d implant procedure, and LV pacing leads positioning}

Experienced electrophysiologists in CRT implantation performed the three CRT leads positioning in cardiac chambers, and then connected to the CRT-d generator. All CRT-d implant procedures were standardized. Right atrial catheters were all placed in right atrial appendage, and right ventricular catheters in right ventricle apex, as indicated by antero-posterior, right anterior, and left anterior oblique views projections at radioscopic imaging. LV epicardial catheters were placed by percutaneous coronary sinus catheterization in a lateral and/or posterior-lateral target vessel $[15,16]$. We randomly chose a multipolar and/or a bipolar LV pacing lead, as described before in the text. After reaching the target left epicardium vessel, we determined the final LV lead position and pacing configuration, by acceptability of pacing thresholds, absence of diaphragmatic stimulation, and anatomic position (chosen position in the target vessel). The final position of the LV pacing lead was assessed with cine fluoroscopy. Implantation duration was defined as the time between skin incision until suture. We used bipolar LV pacing leads (St Jude Medical, Sylmar, CA, USA; Medtronic, Minneapolis, MN, USA), and quadripolar LV pacing leads (Quartet ${ }^{\circledR}$ model $1458 \mathrm{Q}$ and Promote $\mathrm{Q}^{\circledR}$, St Jude Medical, Sylmar, CA, USA; Attain Performa ${ }^{\circledR}$ model, Medtronic, Minneapolis, MN, USA), over-the-wire, steroid eluting with a in-line connector. LV pacing leads were connected to an appropriate bipolar CRT-D device (CRT-d device, St Jude Medical, Sylmar, CA, USA; Medtronic, Minneapolis, MN, USA), and/or to a quadripolar CRT-d device (Quadra Assura CRT-d device, St Jude Medical, Sylmar, CA, USA; Viva ${ }^{\circledR}$ Quad XT and Viva ${ }^{\circledR}$ Quad S cardiac CRT-d, Minneapolis, MN, USA).

\section{Follow up phase \\ Lead functionality parameters}

Right atrium, right ventricle, and left ventricle leads functionality parameters (sensing, impedance, and pacing thresholds) were measured as reported, and indicated by international guidelines $[15,16]$. These parameters were $\mathrm{P}$, and R-wave amplitude values (sensing thresholds), lead impedances values (impedance thresholds), and lead pacing outputs values (pacing thresholds) (Figs. 2, 3). We monitored, measured, and reported these three parameters according to author's suggestions [17]. The sensing thresholds values, defined as $P$ wave and $R$ wave sensing amplitude, were obtained from the intra-cardiac electrograms records, measured using a sensing configuration $[18,19]$. The pacing thresholds, and impedance thresholds values, were measured using pacing catheter configurations $[18,19]$. To measure intra thoracic impedance (Ohm), and pacing thresholds (Volt for ms), we focused on the right ventricle (RV) coil electrode to device case pathway configuration, and on the left ventricle (LV) tip to LV ring configuration $[18,19]$.

\footnotetext{
(See figure on next page.)

Fig. 2 In this figure the representation of cumulative survival events free curves for study endpoints, by Cox regression analysis curves. The figure is structured in seven parts, as a-f, Fig. 3. In green color the bipolar group, in blue color the multipolar group for each figure part. The symbol asterisk was marking a statistical significant event, as indicated by a p value $<0.05$. In the part $\mathbf{a}$ of the figure, the curve representation of Phrenic Nerve stimulation events as "cumulative risk for Phrenic Nerve stimulation" (on y axis) during 360 days follow up (on x axis) comparing multipolar vs bipolar group. In the part b of the figure, the curve representation of catheter dislocation events as "cumulative risk for catheter dislodgement events" (on y axis) during 360 days follow up (on x axis) comparing multipolar vs bipolar group. In the part c of the figure, the curve representation of re-interventions for left ventricle lead re-positioning after dislodgment as "cumulative risk for re-interventions for left ventricle catheter re-positioning" (on y axis) during 360 days follow up (on $x$ axis) comparing multipolar vs bipolar group. In the part $\mathbf{d}$ of the figure, the curve representation of hospital admission events as "cumulative risk for hospital admissions events" (on y axis) during 360 days follow up (on x axis) comparing multipolar vs bipolar group. In the part e of the figure, the curve representation of atrial fibrillation events as "cumulative risk for atrial fibrillation events" (on y axis) during 360 days follow up (on $x$ axis) comparing multipolar vs bipolar group. In the part $\mathbf{f}$ of the curve, the representation of all cause of deaths events as "cumulative risk for all cause of deaths events" (on y axis) during 360 days follow up (on x axis) comparing multipolar vs bipolar group
} 


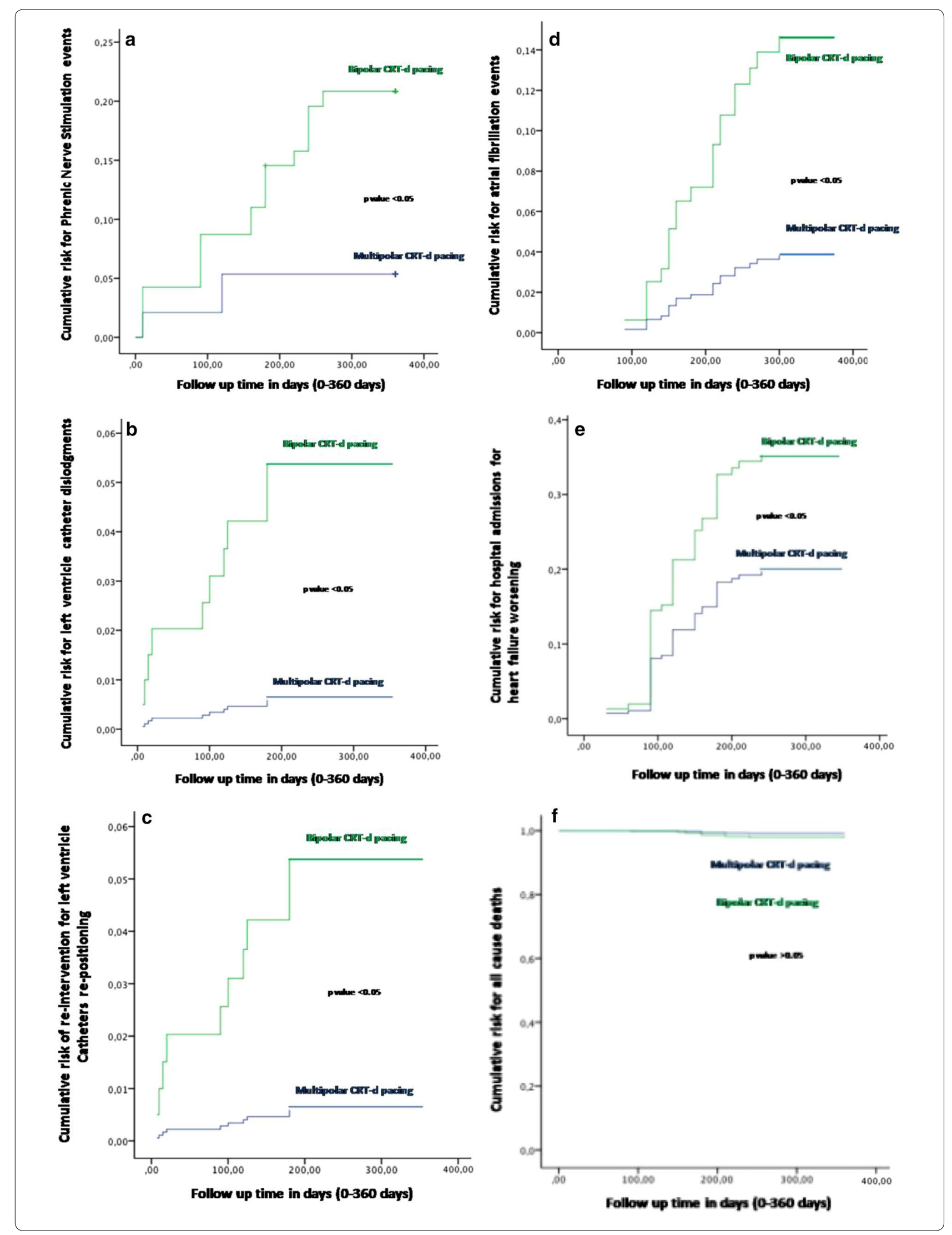




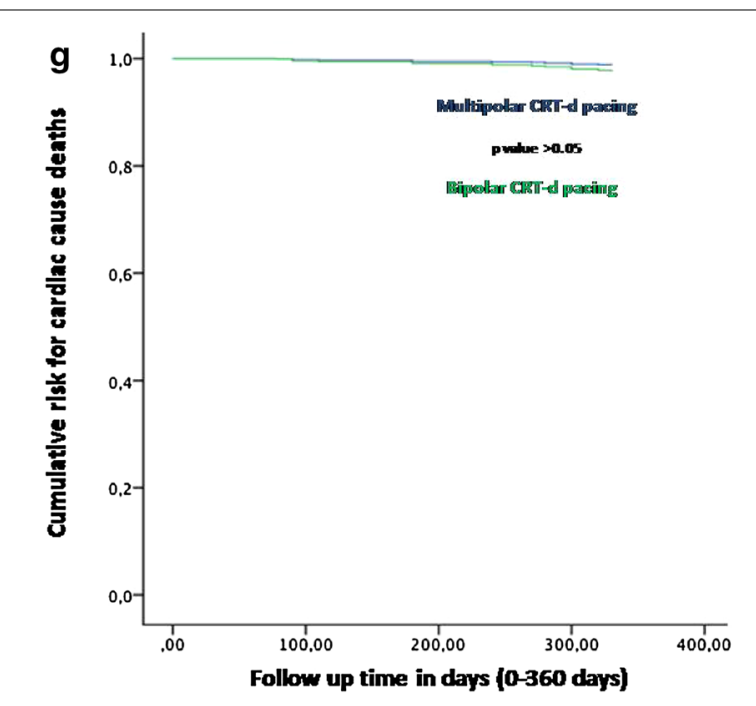

Fig. 3 In this figure the representation of cumulative survival events free curves for study endpoints, by Cox regression analysis curves. In the part of the curve, the representation of cardiac deaths events as "cumulative risk for cardiac deaths events" (on y axis) during 360 days follow up (on x axis) comparing multipolar vs bipolar group

\section{Arrhythmic events in CRT-d recipients}

In CRT-d patients we monitored and reported atrial fibrillation (AF), ventricular tachycardia (VT), and ventricular fibrillation (VF) episodes, and implantable cardioverter defibrillator (ICD) shocks. AF was defined as paroxysmal, and/or not paroxysmal according to authors' suggestions [20]. VT was defined as arrhythmia originating from ventricular chambers, sustained and/or not sustained by arrhythmic event duration [21].VF was defined as a fibrillating arrhythmia originating from ventricular chambers, and associated to hemodynamic instability, and cardiac arrest [21]. ICD shocks were defined as high energy interventions by CRT-d device to restore sinus rhythm during at risk of life sustained VT and or VF events $[15,16]$.

\section{Phrenic nerve stimulation events}

PNS and left ventricle pacing leads threshold were measured by a standard protocol at CRT-d implant, and during all follow up durations by CRT-d devices interrogations. We evaluated these parameters according to authors' suggestions, and previous experiences [22]. We tested PNS thresholds during respiratory phases, and supine, left lateral, right lateral, sitting, and standing body position, in each follow up phase, and for every patient. We accepted the pacing configuration (multipolar and bipolar CRT-d pacing) testing the absence of PNS at pacing output of $7.5 \mathrm{~V}$, with a measured pacing threshold less than or equal to $2.5 \mathrm{~V}$. In case of PNS diagnosis during follow up, by patients symptoms assessment, devices interrogations, and physician ambulatory diagnosis (clinical and instrumental PNS diagnosis), we reached the best left ventricle lead pacing configuration to solve PNS. This test was repeated in both clinical group (multipolar vs bipolar LV pacing lead) of CRT-d patients. In this way we reached the final biventricular pacing configuration by LV pacing lead configuration at best pacing thresholds, and in absence of PNS, and after evaluating the effects of different configurations on hemodynamic parameters, as indicated by authors [22].

\section{LV leads dislodgments}

LV catheter dislodgments resulted by the movement of the catheter into and out of the coronary sinus implantation vessel site, and then causing a change in the catheter tip location [22]. LV catheter dislodgments event was diagnosed by patients clinical symptoms, hospital admissions schedules, hospital discharge schedules, and during medical interrogation at follow up visits. It resulted in altered sensing, pacing, and impedance thresholds of LV lead at device interrogations during follow up. LV catheter dislodgment was confirmed by radiographic biplane projections assessment [22].

\section{CRT-d effect on clinical outcomes (interventions for LV lead re-positioning, CRT responders rate, and clinical events)}

$C R T-d$ responders rate was evaluated by periodic clinical examination, and echocardiography assessment $[15,16]$. Interventions for LV lead re-positioning were defined as interventions done after the first CRT-d implant [22], and were evaluated by hospital admissions schedules, hospital discharge schedules, and during medical interrogation at follow up visits. These interventions were performed in case of LV catheter dislodgments. As other clinical outcomes, hospitalization rate was reported during telephonic interviews, by hospital admissions schedules, hospital discharge schedules, and during medical interrogation at follow up visits. Cardiac deaths, all cause of deaths, and stroke events were evaluated during office follow up visits 10 days after clinical discharge, and after 6th and 12th months by the treating physician, by telephonic interview, hospital admission, and discharge schedules. At each clinical follow-up, right atrial, right ventricular, and left ventricular leads functionality, atrial, and ventricular arrhythmias, ICD shocks, and biventricular pacing percentage, were evaluated and reported for each patient. NYHA classification was re-assessed, and patients graded their overall condition as unchanged or slightly, moderately, or markedly worsened, or improved since randomization by global self-assessment [23]. All patients were instructed to report about devices alarms, loss of lead capture, phrenic nerve stimulation, and arrhythmias. All patients were instructed regularly to 
assess body weight, occurrence of dyspnea, and any clinical symptom. At each visit patients were asked whether medical events or symptoms suggestive of cardiac arrhythmias occurred, and an ECG, and an ECG Holter monitoring, were both performed to detect the presence of asymptomatic arrhythmias. Clinical evaluations included physical examination, vital signs, and review of adverse events. A fasting blood (at least $12 \mathrm{~h}$ from last meal) was performed for biochemical peripheral blood assay evaluation at every visit.

\section{Study endpoints}

As primary endpoints we monitored CRT-d effect in multipolar CRT-d patients vs bipolar CRT-d patients in terms of PNS episodes, LV leads dislodgments, interventions to re-position LV leads, hospitalization rate for HF worsening, cardiac deaths, and all cause deaths. As secondary endpoints we monitored CRT-d responders rate, AF events, VT events, VF events, ICD shocks, and strokes in both groups (multipolar CRT-d vs bipolar CRT-d patients).

\section{Statistical methods}

A qualified statistician analyzed all collected data. The patients were divided before in multipolar CRT-d group vs bipolar CRT-d group, and during follow up visits, and controls in CRT-d responders vs CRT-d non-responders. We postulated that, the number of patients with alterations in primary and secondary endpoints was significantly different between multipolar CRT-d patients vs bipolar CRT-d patients. Safety analyses were performed on data from all enrolled patients. Continuous variables were expressed as means and standard deviations, and were tested by two-tailed Student $t$ test for paired or unpaired data, as appropriate, or by one-way analysis of variance (ANOVA) for more than two independent groups of data. The categorical variables were compared by Chi square or Fisher exact test where appropriate. Survival analysis was performed with the use of the Kaplan-Meier method. Predictors of the study endpoints were evaluated by using Cox regression models. A univariate analysis was conducted to examine the association between single principal clinic, echocardiographic, electrocardiographic characteristics, and multipolar LV pacing, and 12 months study outcomes, as catheter dislodgments, PNS events, re-intervention for catheter dislodgments, hospital admissions for heart failure worsening, and AF events. All variables with $p$ value of less than 0.2 in the univariate analysis were subsequently entered into a multivariate model. In the multivariate model, variables were separately selected and a $p$ value of less than 0.05 was considered significant. For all independent predictors, 95\% confidence intervals were calculated. Statistical significance was considered for a $\mathrm{p}$ value of less than 0.05 . The statistical analysis was performed using the SPSS software package for Windows 17.0 (SPSS Inc., Chicago Illinois).

\section{Results}

One hundred and ninety-five T2DM failing heart patients treated by a CRT-d completed the study follow up, 99 multipolar vs 95 bipolar CRT-d patients (Fig. 1). Mean population age was $67.4 \pm 6.5$ years in overall population, $68.1 \pm 6.6$ vs $66.9 \pm 6.4$ years comparing multipolar vs bipolar CRT-d patients ( $\mathrm{p}$ value $>0.05$ ) (Table 1). One hundred and forty-four patients $(74 \%)$ in overall population, and $71(72 \%)$ vs $73(76 \%)$ patients comparing multipolar vs bipolar CRT-d patients were males ( $\mathrm{p}$ value $>0.05$ ) (Table 1). Other clinical characteristics, echocardiographic parameters, and drug therapy at enrolment were similar, and balanced between two groups of patients (Table 1). Not differently from these results, we reported similar procedural data as skin to skin time $(183 \pm 113$ vs $179 \pm 116 \mathrm{~min}$, $\mathrm{p}$ value $>0.05)$, fluoro time $(17 \pm 4.7$ vs $18 \pm 5.6 \mathrm{~min}, \mathrm{p}$ value $>0.05)$, and CS cannulation time $(14 \pm 5.4$ vs $15 \pm 4.7 \mathrm{~min}, \mathrm{p}$ value $>0.05$ ), and CRT-d leads functionality parameters (left ventricle, right atrial, and right ventricular leads) comparing multipolar vs bipolar CRT-d patients (Table 2). During follow up, primary and secondary study endpoints were reported in study population, comparing multipolar to bipolar CRT-d patients (Table 3). At follow up, there was a statistical significant difference comparing multipolar to bipolar CRT-d patients, about phrenic nerve stimulation events [5 (5\%) vs 18 (18.7\%), p value 0.007], catheter displacement events [ 1 (1\%) vs 9 (9.4\%), p value 0018], and reinterventions for left leads re-positioning [1 (1\%) vs 9 (9.4\%), p value 0.018$]$, hospitalizations for heart failure worsening [15 (15.2\% vs $24(25 \%)$, p value 0.046 ], and atrial fibrillation events [7 (7\%) vs 16 (16.7\%), p value 0.019] (Table 3). At multivariate analysis, quadripolar LV lead vs bipolar LV lead pacing was associated to a reduction of LV catheter dislodgments in a percentage of more than 88\% (HR 0.112 [0.014-0.893], 95\% CI, p value 0.039$)$, and of $75 \%$ for phrenic nerve stimulations events (HR 0.246, [0.088-0.686], 95\% CI, p value 0.007) (Table 4). Multipolar LV pacing reduced at more than $88 \%$ the cases of re-interventions for LV lead repositioning (HR 0.112 [0.014-0.893], 95\% CI, p value 0.039) (Table 4). Multipolar LV lead vs bipolar LV pacing was associated to a reduction of hospital admissions for heart failure worsening in a percentage more than $48 \%$ (HR 0.516, [0.279-0.955], 95\% CI, p value 0.035) 
Table 1 Baseline data of the study cohort (clinical characteristics, echocardiographic parameters, and drug therapy)

\begin{tabular}{|c|c|c|c|c|}
\hline Variables & Overall & Multipolar group & Bipolar group & $p$ value \\
\hline \multicolumn{5}{|l|}{ Clinical characteristics } \\
\hline Patients n & 195 & 99 & 96 & \\
\hline Age, years & $67.4 \pm 6.5$ & $68.1 \pm 6.6$ & $66.9 \pm 6.4$ & n.s \\
\hline Male, n (\%) & $144(74 \%)$ & $71(72 \%)$ & $73(76 \%)$ & n.s \\
\hline Smokers (\%) & $102(52.3 \%)$ & $52(52.5 \%)$ & $50(52 \%)$ & n.s \\
\hline Dyslipidemia (\%) & $102(52 \%)$ & $52(53)$ & $50(52)$ & n.s \\
\hline Obesity & $12(6.1 \%)$ & $7(7 \%)$ & $5(5.2 \%)$ & n.s \\
\hline Hypertension & $136(69.7 \%)$ & $71(71.7 \%)$ & $65(67.7 \%)$ & n.s \\
\hline Renal insufficiency (\%) & $18(9.2 \%)$ & $10(10.1 \%)$ & $8(8.3 \%)$ & n.s \\
\hline Ischemic HF (\%) & $128(65.6 \%)$ & $67(68 \%)$ & $61(63.5 \%)$ & n.s \\
\hline Non ischemic HF (idiopathic, hypertensive, or valvular) (\%) & $67(34.4 \%)$ & $32(32.2 \%)$ & 35 (36.4\%) & n.s \\
\hline Previous cardiac surgery (\%) & $35(18 \%)$ & $17(18 \%)$ & $18(19 \%)$ & n.s \\
\hline NYHA II (\%) & $102(52.3 \%)$ & $53(53.5 \%)$ & $50(52 \%)$ & n.s \\
\hline NYHA III (\%) & $93(47.7 \%)$ & $49(49.5 \%)$ & $44(45.8 \%)$ & n.s \\
\hline QRS duration & $136.4 \pm 7.8$ & $137.3 \pm 7.4$ & $135.3 \pm 8.1$ & n.s \\
\hline $6 \mathrm{MWT}$ & $244.1 \pm 39.8$ & $239.5 \pm 44.7$ & $248.7 \pm 33.8$ & n.s \\
\hline NT-proBNP (pg/ml) & $2307 \pm 631$ & $2322 \pm 567$ & $2281 \pm 723$ & n.s \\
\hline $\mathrm{Hb} 1 \mathrm{Ac}(\mathrm{mmol} / \mathrm{mol})$ & $57.8 \pm 15.6$ & $57.2 \pm 15.3$ & $58.2 \pm 16.1$ & n.s \\
\hline \multicolumn{5}{|l|}{ Echocardiographic parameters } \\
\hline LVEDV (ml) & $198 \pm 39$ & $196 \pm 31$ & $201 \pm 45$ & n.s \\
\hline LVESV $(\mathrm{ml})$ & $137 \pm 29$ & $135 \pm 23$ & $141 \pm 36$ & n.s \\
\hline LVEF (\%) & $27 \pm 5$ & $27 \pm 5$ & $28 \pm 4$ & n.s \\
\hline \multicolumn{5}{|l|}{ Mitral regurgitation } \\
\hline+ & $91(46.7 \%)$ & $44(44.4 \%)$ & $47(49 \%)$ & n.s \\
\hline++ & $77(39.5 \%)$ & $40(40.4 \%)$ & 37 (38.5\%) & n.s \\
\hline+++ & $19(9.7 \%)$ & $9(9.1 \%)$ & $10(10.4 \%)$ & n.s \\
\hline \multicolumn{5}{|l|}{ Drug therapy } \\
\hline ACE-i/ARB & $170(87.2 \%)$ & $86(86.8 \%)$ & $84(87.5 \%)$ & n.s \\
\hline Beta blockers & $137(70.3 \%)$ & $70(71 \%)$ & $67(69.8 \%)$ & n.s \\
\hline Diuretics & $117(60 \%)$ & $58(58.6 \%)$ & $59(61.5 \%)$ & n.s \\
\hline Digoxin & $43(22 \%)$ & $21(21.2 \%)$ & $22(22.9 \%)$ & n.s \\
\hline Statins & $113(57.9 \%)$ & $56(56.6 \%)$ & $57(59.4 \%)$ & n.s \\
\hline Insulin & $72(36.9 \%)$ & $35(35.3 \%)$ & 37 (38.5\%) & n.s \\
\hline Oral hypoglycemic drugs & $131(67.2 \%)$ & $68(68.7 \%)$ & $63(66 \%)$ & n.s \\
\hline Anti platelets drugs & $130(66.7 \%)$ & $64(63.6 \%)$ & $65(67.7 \%)$ & n.s \\
\hline Dicumarolic anticoagulants & $18(9.2 \%)$ & $9(9 \%)$ & $9(9.4 \%)$ & n.s \\
\hline
\end{tabular}

In this table clinical characteristics, drug therapy and echocardiographic parameters have been reported, at baseline, of overall population, and then comparing multipolar vs bipolar group of patients. Statistical analysis has been conducted, to compare categorical data, with the exact Pearson's $\mathrm{X}^{2}$ test. We considered a twosided $p$ value of less than 0.05 as statistically significant. The " $n . s$ " was for statistical not significant ( $p$ value $>0.05$ )

$A C E-i$ angiotensin converting enzyme inhibitor, $A R B$ angiotensin receptor II blockers, COPD chronic obstructive pulmonary diseases, $H b 1 A c$ glycosylated hemoglobin, $y$ year, $n$ number, LVEDv left ventricle end diastolic volume, LVESv left ventricle end systolic volume, LVEF left ventricle ejection fraction, $6 M W T 6$ min walking test, $\mathrm{n}$ is for number, NYHA New York Hearth Association, NOACs new oral anti coagulations drugs, n.sis not statistical significant ( $\mathrm{p}$ value $>0.05$ ), NT-proBNP N terminal pro B type Natriuretic peptide, in mitral regurgitation the symbol,,++++++ indicating low grade $(+)$, mild grade $(++)$, and more than mild $(+++)$ regurgitation grade

(Table 4). Multipolar LV lead pacing was associated to a reduction of atrial fibrillation events in a percentage of more than $73 \%$ (HR 0.261 [0.086-0.794], 95\% CI, p value 0.018) (Table 4). On the contrary, obesity was associated to increased risk to have atrial fibrillation events (HR 1.36, [1.09-1.88], 95\% CI, p value 0.02) (Table 4).

\section{Discussion}

In our study the multipolar CRT-d pacing was found to be superior to the bipolar CRT-d pacing respect to the reduction of PNS events, LV leads dislodgment, re-interventions for LV leads dislodgments, hospitalizations for HF worsening, and AF events (Figs. 2, 3; Table 3). The 
Table 2 Procedural data of the study cohort

\begin{tabular}{|c|c|c|c|}
\hline Parameters & Quadripolar group (n 99) & Bipolar group (n 96) & p value \\
\hline Skin to skin time & $183 \pm 113$ & $179 \pm 116$ & n.s \\
\hline Fluoro time & $17 \pm 4.7$ & $18 \pm 5.6$ & n.s \\
\hline CS cannulation time & $14 \pm 5.4$ & $15 \pm 4.7$ & n.s \\
\hline \multicolumn{4}{|l|}{ CS target vessel } \\
\hline Anterior, n (\%) & $3(3)$ & $3(3)$ & n.s \\
\hline Anterolateral, n (\%) & $13(13)$ & $11(12)$ & n.s \\
\hline Lateral, n (\%) & $51(51)$ & $52(54)$ & n.s \\
\hline Postero-lateral, n (\%) & $28(28)$ & $25(26)$ & n.s \\
\hline Posterior, n (\%) & $4(4)$ & $5(5)$ & n.s \\
\hline \multicolumn{4}{|l|}{ LV lead parameters } \\
\hline Impedance thresholds & $761 \pm 182$ & $685 \pm 196$ & n.s \\
\hline Pacing thresholds & $0.5 \pm 0.4$ & $0.5 \pm 0.3$ & n.s \\
\hline Sensing thresholds & $14 \pm 7.2$ & $13.4 \pm 7.3$ & n.s \\
\hline \multicolumn{4}{|l|}{ RA lead parameters } \\
\hline Impedance thresholds & $438 \pm 164$ & $452 \pm 133$ & n.s \\
\hline Pacing thresholds & $0.4 \pm 0.2$ & $0.5 \pm 0.3$ & n.s \\
\hline Sensing thresholds & $2.1 \pm 1.7$ & $2.3 \pm 1.8$ & n.s \\
\hline \multicolumn{4}{|l|}{ RV lead parameters } \\
\hline Impedance thresholds & $581 \pm 151$ & $577 \pm 163$ & n.s \\
\hline Shock impedance thresholds & $73 \pm 15$ & $76 \pm 14$ & n.s \\
\hline Pacing thresholds & $0.5 \pm 0.45$ & $0.43 \pm 0.35$ & n.s \\
\hline Sensing thresholds & $19 \pm 6$ & $18 \pm 5$ & n.s \\
\hline
\end{tabular}

Table 3 Clinical events of the study cohort

\begin{tabular}{|c|c|c|c|c|}
\hline Clinical events & Overall (n 195) & Quadripolar group (n 99) & Bipolar group (n 96) & p value \\
\hline CRT-d responders rate (\%) & $117(60 \%)$ & $61(61.6 \%)$ & $56(58 \%)$ & 0.27 \\
\hline Phrenic nerve stimulation & $23(11.8 \%)$ & $5(5 \%)$ & $18(18.7 \%)$ & $0.007^{*}$ \\
\hline Catheter displacement & $10(5.1 \%)$ & $1(1 \%)$ & $9(9.4 \%)$ & $0.018^{*}$ \\
\hline Re-interventions & $10(5.1 \%)$ & $1(1 \%)$ & $9(9.4 \%)$ & $0.018^{*}$ \\
\hline Hospitalizations for HF worsening & $39(20 \%)$ & $15(15.2 \%)$ & $24(25 \%)$ & $0.046^{*}$ \\
\hline Stroke & $4(2 \%)$ & $2(2 \%)$ & $2(2 \%)$ & 0.62 \\
\hline$A F n$ & $21(10.8 \%)$ & $7(7 \%)$ & $16(16.7 \%)$ & $0.019^{*}$ \\
\hline VTn & $51(26.1 \%)$ & $25(25.2 \%)$ & $26(27.1 \%)$ & 0.5 \\
\hline ICD shocks & $27(13.8 \%)$ & $13(13.1 \%)$ & $14(14.6 \%)$ & 0.51 \\
\hline Cardiac deaths & $9(5.6 \%)$ & $4(4 \%)$ & $5(5.2 \%)$ & 0.43 \\
\hline All cause deaths & $12(6.2 \%)$ & $5(5 \%)$ & $7(7.3 \%)$ & 0.33 \\
\hline Stroke & $5(2.6 \%)$ & $2(2 \%)$ & $3(3.1 \%)$ & 0.36 \\
\hline
\end{tabular}

In this table are reported clinical events after the CRT-d implant in quadripolar vs bipolar group

$A F$ atrial fibrillation, $C R T-d$ cardiac resynchronization therapy with a defibrillator, $H F$ heart failure, ICD internal cardioverter defibrillator, $V T$ ventricular tachycardia The symbol * was marking a statistical significant value, as $p$ value $<0.05$

analysis of the others study endpoints did not show differences between the two study groups with regard to cardiac deaths, all cause deaths, strokes, VT events, and
ICDs shocks, and CRT-d responders rate (Table 3). In literature less has been reported about the effect of diabetes mellitus on the risks of arrhythmias, and ICDs therapies 
Table 4 Univariate and multivariate analysis of predictive factors of the study cohort outcomes

\begin{tabular}{|c|c|c|c|c|c|c|}
\hline & HR & Univariate $(95 \% \mathrm{Cl})$ & $p$ value & HR & Multivariate $(95 \% \mathrm{Cl})$ & $p$ value \\
\hline \multicolumn{7}{|c|}{ A. Catheter dislodgments } \\
\hline Age & 0.902 & {$[0.807-1.008]$} & 0.068 & 0.866 & {$[0.741-1.013]$} & 0.072 \\
\hline Obesity & 1.718 & {$[0.218-3.568]$} & 0.608 & & & \\
\hline Renal dysfunction & 1.073 & {$[0.136-4.873]$} & 0.946 & & & \\
\hline NYHA 3 & 0.417 & [0.108-1.612] & 0.205 & & & \\
\hline QRS duration & 0.962 & {$[0.872-1.061]$} & 0.437 & & & \\
\hline LVEF & 1.072 & {$[0.928-1.237]$} & 0.345 & & & \\
\hline Quadripolar LV & 5.278 & {$[1.175-7.32]$} & $0.035^{*}$ & 0.112 & [0.014-0.893] & $0.039^{*}$ \\
\hline \multicolumn{7}{|c|}{ B. Phrenic nerve stimulation } \\
\hline Age & 1.024 & [0.962-1.089] & 0.458 & & & \\
\hline Obesity & 2.604 & {$[0.773-8.767]$} & 0.122 & 0.658 & {$[0.144-3.01]$} & 0.59 \\
\hline Renal dysfunction & 0.043 & {$[0.02-1.79]$} & 0.306 & & & \\
\hline NYHA 3 & 2.94 & {$[2.6-4.9]$} & $0.001^{*}$ & 0.97 & {$[0.022-0.423]$} & 0.2 \\
\hline QRS duration & 1.019 & {$[0.975-1.065]$} & 0.405 & & & \\
\hline LVEF & 1.012 & {$[0.93-1.101]$} & 0.79 & & & \\
\hline Quadripolar LV & 3.783 & [1.404-10.191] & $0.008^{*}$ & 0.246 & [0.088-0.686] & $0.007^{*}$ \\
\hline \multicolumn{7}{|c|}{ C. Re-intervention for catheter dislodgments } \\
\hline Age & 0.902 & {$[0.807-1.008]$} & 0.068 & 0.866 & {$[0.741-1.013]$} & 0.072 \\
\hline Obesity & 1.718 & {$[0.218-3.568]$} & & & & \\
\hline Renal dysfunction & 1.073 & {$[0.136-4.873]$} & & & & \\
\hline NYHA 3 & 0.417 & {$[0.108-1.612]$} & & & & \\
\hline QRS duration & 0.962 & {$[0.872-1.061]$} & & & & \\
\hline LVEF & 1.072 & {$[0.928-1.237]$} & & & & \\
\hline Quadripolar LV & 5.278 & {$[1.175-7.32]$} & $0.035^{*}$ & 0.112 & {$[0.014-0.893]$} & $0.039^{*}$ \\
\hline \multicolumn{7}{|c|}{ D. Hospital admission for heart failure worsening } \\
\hline Age & 0.823 & {$[0.961-1.051]$} & 0.823 & & & \\
\hline Obesity & 1.062 & {$[0.33-3.419]$} & 0.92 & & & \\
\hline Renal dysfunction & 0.898 & {$[0.322-2.501]$} & 0.836 & & & \\
\hline NYHA 3 & 1.667 & {$[0.926-3.01]$} & 0.089 & 0.624 & [0.326-1.193] & 0.154 \\
\hline QRS duration & 1.019 & {$[0.983-1.056]$} & 0.3 & 1.012 & {$[0.973-1.052]$} & 0.559 \\
\hline LVEF & 0.98 & [0.926-1.038] & & & & \\
\hline Quadripolar LV & 1.683 & {$[1.04-3.03]$} & 0.05 & 0.516 & [0.279-0.955] & $0.035^{*}$ \\
\hline \multicolumn{7}{|c|}{ E. Atrial fibrillation events } \\
\hline Age & 1.031 & [0.968-1.099] & 0.343 & & & \\
\hline Obesity & 5.571 & [2.038-15.232] & $0.001^{*}$ & 1.36 & {$[1.09-1.88]$} & $0.02^{*}$ \\
\hline Renal dysfunction & 0.992 & {$[0.231-4.257]$} & 0.991 & & & \\
\hline NYHA 3 & 2.58 & [1.001-6.649] & $0.05^{*}$ & 0.557 & [0.195-1.591] & 0.274 \\
\hline QRS duration & 1.018 & {$[0.971-1.067]$} & 0.457 & & & \\
\hline LVEF & 1.043 & {$[0.95-1.146]$} & 0.375 & & & \\
\hline Quadripolar LV & 3.29 & [1.205-8.982] & $0.02^{*}$ & 0.261 & {$[0.086-0.794]$} & $0.018^{*}$ \\
\hline \multicolumn{7}{|l|}{ F. All cause deaths } \\
\hline Age & 1.138 & {$[1.04-1.246]$} & $0.05^{*}$ & 1.149 & {$[0.89-1.288]$} & 0.058 \\
\hline Obesity & 2.5 & {$[0.9-2.91]$} & 0.986 & & & \\
\hline Renal dysfunction & 0.88 & [0.108-7.193] & 0.905 & & & \\
\hline NYHA 3 & 1.471 & {$[0.276-7.759]$} & 0.649 & & & \\
\hline QRS duration & 1.051 & [0.984-1.123] & 0.141 & & & \\
\hline LVEF & 1.069 & [0.914-1.249] & 0.404 & & & \\
\hline Quadripolar LV & 0.415 & [0.104-1.659] & 0.214 & & & \\
\hline
\end{tabular}


Table 4 continued

\begin{tabular}{|c|c|c|c|c|c|c|}
\hline & HR & Univariate $(95 \% \mathrm{Cl})$ & $p$ value & HR & Multivariate $(95 \% \mathrm{Cl})$ & $p$ value \\
\hline \multicolumn{7}{|l|}{ G. Cardiac deaths } \\
\hline Age & 1.3 & [1.185-1.426] & $0.01^{*}$ & 1.332 & [0.858-1.532] & 0.056 \\
\hline Obesity & 0.7 & {$[0.2-2.896]$} & 0.168 & & & \\
\hline Renal dysfunction & 1.676 & {$[0.2-14.02]$} & 0.633 & & & \\
\hline NYHA 3 & 0.386 & {$[0.069-2.164]$} & 0.279 & & & \\
\hline QRS duration & 0.932 & {$[0.806-1.078]$} & 0.342 & & & \\
\hline LVEF & 0.912 & {$[0.815-1.044]$} & 0.201 & & & \\
\hline Quadripolar LV & 0.521 & [0.154-1.758] & 0.293 & & & \\
\hline
\end{tabular}

In this table the representation of study outcomes, as catheter dislodgments (A), phrenic nerve stimulation events (B), re-intervention for catheter dislodgments (C), hospital admissions for heart failure worsening (D), atrial fibrillation events (E), all cause deaths (F), and cardiac deaths (G), and multivariate predictive factors

We have used for statistical analysis, a $95 \%$ interval of confidence (IC), and a significant statistical $p$ value, $p<0.05$. The symbol * was marking factor with a $p$ value $<0.05$. To test the final statistical used model, we have performed the Hosmer and Lemeshow test, with a $x^{2}=2.775$, and a $p$ value $<0.05$

NYHA 3 New York Heart Association third class, LVEF left ventricle ejection fraction, $L V$ left ventricle

[24]. Moreover, although authors reported a significant excess of cardiac hospitalizations and mortality in the diabetic population, this higher risk is not related to arrhythmias and/or to a difference in the rate of ICD treatments [24]. We reported a statistical significant reduction of PNS events in multipolar group vs bipolar group [n 5 (\%) vs n 18 (18.7\%), p value 0.007] (Table 3; Fig. 2a). PNS is a challenging, and a relevant problem in failing heart patients treated by CRT-d [25-27], reported in a percentage of $7-14 \%$ patients treated by bipolar LV leads, and associated to the LV lead location [14, 28]. In fact, PNS is more common with the LV lead in the midapical, posterior and lateral sites, and less common with the LV lead in the anterior or basal site [14, 28]. Multipolar CRT-d pacing may reduce PNS. Physicians may reach and pace the target coronary vessel, and the target LV wall segment, by a LV pacing from electrodes more far from the tip of the lead, that may be more anatomically closed to phrenic nerve course [25-27]. In our study multipolar lead LV pacing was associated to a reduction of $L V$ leads displacements, and subsequently of re-interventions to re-position LV leads [n 1 (1\%) vs n 9 (9.4\%), p value 0.018 ], as compared to bipolar LV pacing (Table 3; Fig. 2b, c). In the multivariate analysis, multipolar LV lead pacing was associated to a reduction of $L V$ leads displacements, and subsequently of re-interventions to re-position LV lead, in a percentage of more than $88 \%$ (HR 0.112 [0.014-0.893], 95\% CI, p value 0.039) (Table 4; Fig. 2b, c). The ability to perform a CRT- $d$ implant may be related to the possibility to cannulate CS, and to reach the target CS vessel $[15,16]$. Sometimes, the anatomic position of coronary sinus vessel, the angle of origin of the vessel, the vessel size, and others variables may render difficult to select the target vessel, and it may consequently affect the stability of the implanted LV lead $[15,16]$. Other times, the best anatomical, and the consequent more stable position in the target vessel may be related to the position of the tip of LV lead more close to the course of the phrenic nerve, as discussed before, and/or more close to LV segments conditioning worse sensing and pacing thresholds [25-27]. Therefore, a more stable vessel position may sometimes condition the sensing and pacing LV thresholds and programming, while on the contrary the best sensing and pacing LV configuration may not result in the more stable position in the target vessel [25-27]. This may lead to re-positioning of LV lead, in a more proximal position in the target vessel, and/or in a different cardiac vein $[15,16]$. These events may be more common seen in bipolar LV leads as compared to multipolar LV leads, by the position of sensing, and pacing couple of poles in the tip of LV bipolar catheter. This may also condition catheter dislodgments after the implant $[15,16$, 25-30]. Both these conditions may lead to dislodgments, replacements, and re-interventions of LV leads. Multipolar LV pacing leads may offer a greater variety of sensing and pacing configurations from different sites within a coronary vein, as compared to bipolar pacing [30]. Therefore, this may reduce LV dislodgments, and re-interventions, allowing optimal LV lead placement to maximize biventricular pacing at long term follow up [29, 30]. Moreover, we may prefer the more stable anatomic position in the target vessel, without replacing more proximally the LV lead, and/or finding others veins to reach the best sensing and pacing thresholds and programming $[29,30]$. These results were similarly addressed by authors in the overall population of failing heart patients treated by multipolar CRT-d pacing [27]. We may speculate that, the placement of multipolar LV lead in a target LV epicardium vessel, because its anatomical stability, and the possibility to program multiple sensing and pacing thresholds, may result in a stable and continuous CRT-d pacing [26]. Moreover, this may consequently 
impact on AF events, and hospitalizations for HF worsening (Table 3; Fig. 2d, e). In fact, we found that multipolar pacing vs bipolar pacing may lead to a significant reduction of AF events [n 7 (7\%) vs 16 (16.7\%), p value 0.019]. Intriguingly, the use of multipolar LV pacing during CRT-d implant may be predictive of lower AF events at follow up (HR 0.261 [0.086-0.794], 95\% CI, p value 0.018) (Figs. 2, 3; Table 3). This effect CRT-d induced has been discussed by different authors. In the CARE-HF study authors reported that, in sinus rhythm patients the global incidence of AF was similar in medical therapy alone as compared to CRT [31]. On the contrary, others authors reported that CRT-d pacing might be associated with shorter duration of AF events [32]. Therefore, the stable and continuous CRT-d pacing may reduce AF burden [31]. Authors supported this hypothesis by the observation of reversal of left atrial remodeling CRT-d induced, and then associated to shorter duration of AF events during CRT-d pacing [32]. Similarly, same authors showed that $\mathrm{AF}$ occurrence was associated with failing heart worsening [32]. These data are in line with our study results. In our study we reported that the obesity was an independent predictor of AF events (HR 1.36 [1.09-1.88], $95 \% \mathrm{CI}$, p value 0.02 ). As first, obesity may be associated to a hyper activation of inflammatory tone, and cytokines expression [33], and to hyper activation of the sympathetic tone [34]. The hyper activation of the sympathetic tone may render obese patients more vulnerable to proarrhythmic stimuli [34]. These functional alterations may be consequently associated to structural abnormalities, as abnormal visceral fat deposition, which may lead to an augmentation of atrial arrhythmic burden [35]. These alterations may condition AF burden, without impacting on VT burden [36]. On the contrary, the higher stability of multipolar LV lead, and the consequent continuous biventricular pacing in a target segment of LV wall, may reduce AF events. Consequently the obesity did not diminish the clinical benefit of CRT-d to reduce risk for appropriate ICD therapy, as we observed in our study [36].This effect, and the opportunity to program multiple LV pacing configurations, may be induced by multipolar pacing [37]. The multipolar stable, and continuous biventricular pacing, may induce a stabilization effect on cellular membranes, ionic currents, and cardiac cells [37]. This effect, reducing AF episodes PNS, LV lead dislodgments, and re-interventions to re-position LV leads, may be associated to a lowering of hospitalizations for $H F$ worsening in T2DM failing heart patients [n $15(15.2 \%)$ vs 24 (25\%), p value 0.046 ], comparing multipolar pacing vs bipolar pacing (Table 4; Fig. 2d). This may be seen as important study result, and a relevant study message. This result may be seen as a strong data supporting our primary study hypothesis. In fact, it may confirm that, in
T2DM failing heart patients the multipolar LV pacing is an advancement of CRT-d pacing safe to use, and that it may be associated to a significant reduction of hospitalizations for HF worsening. In fact, we observed that the choice and the utilization of a multipolar LV pacing lead during CRT-d implant may be an independent predictor factor of hospitalizations for HF worsening (HR 0.516 [0.279-0.955], $\mathrm{p}$ value 0.035) in T2DM failing heart patients (Table 4; Fig. 2d). CRT-d reduces the risk of heart-failure events in patients with a low ejection fraction and wide QRS complex [38], and this effect has been reported also in diabetic patients [36]. CRT-d is a class 1 indication to reduce hospitalizations for HF worsening in failing heart diabetic patients, and also in failing heart non diabetic patients $[15,16]$. Actually, for the first time in literature we may report that, multipolar pacing vs bipolar pacing may significantly reduce hospitalizations for HF worsening in T2DM failing heart patients. Parallely, we may observe that, the choice of multipolar CRT-d pacing may be predictive of hospitalizations lowering for HF worsening in T2DM failing heart patients. In our study, the reduction of HF events induced by multipolar CRT-d pacing, was not associated to a reduction of the mortality (cardiac deaths, and all cause deaths). Similarly, multipolar CRT-d pacing in T2DM patients did not significantly improve the CRT-d responder's rate. These results have to be seen in the complexity of diabetes, and of its effect on failing heart disease, and on clinical outcomes in CRT-d recipients $[11,12]$. All these results, such as the not significant improvement in the percentage of CRT-d responders, may be related to higher percentage of ischemic cardiac disease conditioning HF in T2DM patients, and then impacting on CRT-d response $[1-4,6-12]$.

\section{Study limitations}

This study had few limitations. As first, we examined a small percentage of T2DM failing heart patients treated by multipolar vs bipolar CRT-d, as compared to overall population. This was due to loss of patients during follow up, and to the low adherence of patients to the study protocol as discussed in results session. Second, this study was conducted at 12 months follow up time, and this short time follow up duration may affect the long term follow up prognosis, and primary and secondary clinical outcomes. Third, we have to report the paucity of clinical characteristics that would provide a more accurate comparison to clinical trial subjects. At last we have not investigated the molecular, and epigenetic aspects induced by T2DM in failing heart patients, and/or modulated by CRT-d. In fact, T2DM by an altered glucose homeostasis may induce electrophysiological changes, which leads to QRS prolongation, decreased conduction 
velocity and increased arrhythmogenesis, and this may condition clinical prognosis [39]. We mentioned data about cardiac electrophysiology properties by CRT devices interrogations, examined, and collected during routine devices interrogations. We did not report data by continuous devices monitoring systems [40]. This may be limiting for our analysis, because continuous monitoring of CRT-d devices may impact positively on clinical outcomes [40]. We did not perform imaging examinations to support cases of left atrial remodeling observed in study population CRT, and associated to shorter duration of AF events as described by authors [30]. In this study we did not perform animal experiments to test these clinical effect induced by multipolar CRT-d pacing in T2DM failing heart subjects, and we loss data about cardiac cells electrical properties, inflammatory tone, oxidative stress modulation, and sympathetic tone activity in T2DM patients. In this study we did not report data about the epigenetic effect CRT-d induced in T2DM failing heart patients, and this may be a limiting factor of our study analysis [41, 42]. In this study we did not compare multipolar Vs bipolar CRT-d pacing in failing heart diabetic vs non diabetic patients.

\section{Conclusion}

In T2DM failing heart patients multipolar CRT-d pacing may reduce PNS, catheters dislodgments, and reinterventions as compared to bipolar CRT-d pacing. This effect may be associated to reduction of AF events, and hospitalizations for HF worsening. Multipolar CRT-d pacing may offer a relevant hemodynamic advantage over a fixed conventional CRT-d pacing by a single site LV lead in failing heart patients [43, 44]. In our study, we focused on these hemodynamic, and clinical effects induced by multipolar CRT-d pacing in a population of T2DM failing heart patients. In fact, in T2DM failing heart patients multipolar LV pacing vs bipolar LV pacing may lead to a statistical significant reduction of hospital admissions for HF worsening. This study endpoint reached by multipolar CRT-d pacing, was not correlated to a statistical significant improvement of CRT-d responders rate in T2DM failing heart patients. Finally, multipolar CRT-d did not affect cardiac deaths, and all cause deaths in a population of T2DM failing heart patients. A part of this, we may speculate that, multipolar CRT-d pacing may represent a possible way to reduce CRT-d failures, and to improve clinical outcomes in T2DM failing heart patients. In fact, multipolar LV pacing may be predictive, as compared to bipolar pacing, of all these adverse procedure related events, and clinical events. We may speculate that, the lowering of AF events, and hospitalizations for HF worsening, and the predictably of these adverse conditions, may lead us to prefer a multipolar vs bipolar pacing in a population of T2DM failing heart patients. Moreover, we may propose multipolar CRT-d pacing as an innovative, and better treatment in T2DM failing heart patients.

\section{Abbreviations}

AF: atrial fibrillation; CRP: C reactive protein; CRT-d: cardiac resynchronization therapy with a defibrillator; HF: heart failure; ICD: implantable cardioverter defibrillator; LV: left ventricle; LVEF: left ventricle ejection fraction; ms: millisecond; mV: millivolt; NYHA: New York Heart Association; PNS: phrenic nerve stimulation; RV: right ventricular; T2DM: type 2 diabetes mellitus; VF: ventricular fibrillation; VT: ventricular tachycardia.

\section{Authors' contributions}

CS wrote the study protocol, collected, analyzed data, performed statistical analysis, and wrote the article. VG and AS collected data. RM and GP revised all the manuscript. All authors read and approved the final manuscript.

\section{Author details}

${ }^{1}$ Department of Medical, Surgical, Neurological, Metabolic and Aging Sciences, University of Campania "Luigi Vanvitelli", Piazza Miraglia, 2, 80138 Naples, Italy. ${ }^{2}$ Cardiovascular and Arrhythmias Department, John Paul II Research and Care Foundation, Campobasso, Italy. ${ }^{3}$ Cardiovascular Department, Inselspital of Bern University, Bern, Switzerland.

Acknowledgements

All authors have equally contributed to the study.

\section{Competing interests}

The authors declare that they have no competing interests.

\section{Availability of data and materials}

Data and materials are available.

\section{Consent for publication}

Authors give full consent for publication of the present article.

\section{Ethics approval and consent to participate}

This study was conducted in accordance with the Declaration of Helsinki. The Ethics Committees of all participating institutions approved the protocol. All patients were informed about the study nature, and gave their written informed, and signed consent to participate in the study.

\section{Funding}

This study was conducted without sponsors and without financial support. C.S. receives a research Grant from European Society of Cardiology.

\section{Publisher's Note}

Springer Nature remains neutral with regard to jurisdictional claims in published maps and institutional affiliations.

Received: 12 May 2017 Accepted: 23 May 2017

Published online: 09 June 2017

\footnotetext{
References

1. Huang ES, Laiteerapong N, Liu JY, John PM, Moffet HH, Karter AJ. Rates of complications and mortality in older patients with diabetes mellitus: the diabetes and aging study. JAMA Intern Med. 2014; 174(2):251-8

2. Devereux RB, Roman MJ, Paranicas M, O'Grady MJ, Lee ET, Welty TK, Fabsitz RR, Robbins D, Rhoades ER, Howard BV. Impact of diabetes on cardiac structure and function: the Strong Heart Study. Circulation. 2000;101:2271-6.

3. Kannel WB, McGee DL. Diabetes and cardiovascular disease: the Framingham study. JAMA. 1979;241:2035-8.
} 
4. Lind M, Bounias I, Olsson M, Gudbjornsdottir S, Svenson AM, Rosengren A. Glycaemic control and incidence of heart failure in 20985 patients with type 1 diabetes: an observational study. Lancet. 2011;378:140-6.

5. Moss AJ, Jackson Hall W, Cannom DS, Klein H, Brown MW, James WS, Daubert P, Mark Estes NA, Foster E, Greenberg H, Higgins SL, Pfeffer MA, Solomon SD, Wilber D, Zareba W, for the MADIT-CRT Trial Investigators*. Cardiac-resynchronization therapy for the prevention of heart-failure events. N Engl J Med. 2009;361:1329-38.

6. Fantoni C, Regoli F, Ghanem A, Raffa S, Klersy C, Sorgente A, Faletra F, Baravelli M, Inglese L, Salerno-Uriarte JA, Klein HU, Moccetti T, Auricchio A. Long-termoutcomein diabetic heart failure patients treated with cardiac resynchronization therapy. Eur J Heart Fail. 2008;10(3):298-307.

7. George J, Barsheshet A, Moss AJ, Martin D, Ouellet G, McNitt S, Goldenberg I. Effectiveness of cardiac resynchronization therapy in diabetic patients with ischemic and nonischemic cardiomyopathy. Ann Non invasive Electrocardiol. 2012;2012(17):14-21.

8. Hoppe UC, Freemantle N, Cleland JGF, Marijianowski M, Erdmann E. Effect of cardiac resynchronization on morbidity and mortality of diabetic patients with severe heart failure. Diabetes Care. 2007;30:722-4.

9. Mangiavacchi M, Gasparini M, Genovese S, Pini D, Klersy C, Bragato R, Andreuzzi B, Municinò A, Regoli F, Galimberti P, Ceriotti C, Gronda E. Insulin-treated type 2 diabetes is associated with a decreased survival in heart failure patients after cardiac resynchronization therapy. Pacing Clin Electrophysiol. 2008;31:1425-32.

10. Ruwald AC, Schuger C, Moss AJ, Kutyifa V, Olshansky B, Greenberg H, Cannom DS, Estes NA, Ruwald MH, Huang DT, Klein H, McNitt S, Beck CA, Goldstein R, Brown MW, Kautzner J, Shoda M, Wilber D, Zareba W, Daubert JP. Mortalityreduction in relation to implantable cardioverter defibrillator programming in the multicenter automatic defibrillator implantation trial-reduce inappropriate therapy (MADIT-RIT). Circ: Arrhythm Electrophysiol. 2014;7(5):785-92.

11. Sardu C, Marfella R, Santulli G. Impact of diabetes mellitus on the clinical response to cardiac resynchronization therapy in elderly people. J Cardiovasc Transl Res. 2014;7(3):362-8.

12. Echouffo-Tcheugui JB, Masoudi FA, Bao H, Spatz ES, Fonarow GC. Diabetes mellitus and outcomes of cardiac resynchronization with implantable cardioverter-defibrillator therapy in older patients with heart failure. Circ Arrhythm Electrophysiol. 2016;9(8):e004132.

13. Boriani G, Connors S, Kalarus Z, Lemke B, Mullens W, Asensi JO, Raatikainen P, Gazzola C, Farazi TG, Leclerca C. Cardiac resynchronization therapy with a quadripolar electrode lead decreases complications at 6 months results of the MORE-CRT randomized trial. JACC: Clin Electrophysiol. 2016;2:2.

14. Osnat G, Eyal N, Shemy C, David L, Bar-Lev D, Nechemya T, Eldar M, Glikson M. Programmable multiple pacing configurations help to overcome high left ventricular pacing thresholds and avoid phrenic nerve stimulation. Pacing Clin Electrophysiol. 2005;28(12):1255-9.

15. Epstein AE, DiMarco JP, Ellenbogen KA, Estes NA 3rd, Freedman RA, Gettes LS, Gillinov AM, Gregoratos G, Hammill SC, Hayes DL, Hlatky MA, Newby LK, Page RL, Schoenfeld MH, Silka MJ, Stevenson LW, Sweeney MO, Tracy CM, Epstein AE, Darbar D, DiMarco JP, Dunbar SB, Estes NA 3rd, Ferguson TB Jr, Hammill SC, Karasik PE, Link MS, Marine JE, Schoenfeld MH, Shanker AJ, Silka MJ, Stevenson LW, Stevenson WG, Varosy PD. American College of Cardiology Foundation; American Heart Association Task Force on Practice Guidelines.; HeartRhythm Society. 2012 ACCF/AHA/HRS Focused Update Incorporated Into the ACCF/AHA/HRS 2008 guidelines for device-based therapy of cardiac rhythm abnormalities. J Am Coll Cardiol. 2013;61 (2):e1-6.

16. Brignole $M$, Auricchio A, Baron-Esquivias $G$, Bordachar $P$, Boriani $G$, Breithardt OA, Cleland J, Deharo JC, Delgado V, Elliott PM, Gorenek B, Israel CW, Leclercq C, Linde C, Mont L, Padeletti L, Sutton R, Vardas PE, ESC Committee for Practice Guidelines (CPG), Zamorano JL, Achenbach S, Baumgartner H, Bax JJ, Bueno H, Dean V, Deaton C, Erol C, Fagard R, Ferrari R, Hasdai D, Hoes AW, Kirchhof P, Knuuti J, Kolh P, Lancellotti P, Linhart A, Nihoyannopoulos P, Piepoli MF, Ponikowski P, Sirnes PA, Tamargo JL, Tendera M, Torbicki A, Wijns W, Windecker S, Document Reviewers, Kirchhof P, Blomstrom-Lundqvist C, Badano LP, Aliyev F, Bänsch D, Baumgartner H, Bsata W, Buser P, Charron P, Daubert JC, Dobreanu D, Faerestrand S, Hasdai D, Hoes AW, Le Heuzey JY, Mavrakis H, McDonagh T, Merino JL, Nawar MM, Nielsen JC, Pieske B, Poposka L, Ruschitzka F, Tendera M, Van Gelder IC, Wilson CM. 2013 ESC guidelines on cardiac pacing and cardiac resynchronization therapy The Task Force on cardiac pacing and resynchronization therapy of the European Society of Cardiology (ESC). Developed in collaboration with the European Heart Rhythm Association (EHRA). Eur Heart J. 2013;34:2281-329.

17. Maisel WH. Physician management of pacemaker and implantable cardioverter defibrillator advisories. Pacing Clin Electrophysiol. 2004;27:437-42.

18. Yu CM, Wang L, Chau E, Chan RH, Kong SL, Tang MO, Christensen J, Stadler RW, Lau CP. Intrathoracic impedance monitoring in patients with heart failure correlation with fluid status and feasibility of early warning preceding hospitalization. Circulation. 2005;112:841-8.

19. Prochazka A, Korpas D. Monitoring biventricular pacing parameters dependingon the left ventricle lead configuration. Exp Clin Cardiol. 2013;18(2):85-8.

20. January CT, Wann LS, Alpert JS, Calkins H, Cigarroa JE, Cleveland JC Jr, Conti JB, Ellinor PT, Ezekowitz MD, Field ME, Murray KT, Sacco RL, Stevenson WG, Tchou PJ, Tracy CM, Yancy CW, American College of Cardiology/ American Heart Association Task Force on Practice Guidelines. 2014 ACC/ AHA/ESC Guidelines for the management of patients with atrial fibrillation: executive summary a report of the American College of Cardiology/ American Heart Association Task Force on Practice Guidelines and the European Society of Cardiology Committee for Practice Guidelines and Policy Conferences (Committee to Develop Guidelines for the Management of Patients With Atrial Fibrillation) Developed in Collaboration With the North American Society of Pacing and Electrophysiology. J Am Coll Cardiol. 2014;64(21):e1-76.

21. Priori SG, Blomström-Lundqvist C, Mazzanti A, Blom N, Borggrefe M, Camm J, Elliott PM, Fitzsimons D, Hatala R, Hindricks G, Kirchhof P, Kjeldsen K, Kuck KH, Hernandez-Madrid A, Nikolaou N, Norekvål TM, Spaulding C, Van Veldhuisen DJ. 2015 ESC Guidelines for the management of patients with ventricular arrhythmias and the prevention of sudden cardiac death: The Task Force for the Management of Patients with Ventricular Arrhythmias and the Prevention of Sudden Cardiac Death of the European Society of Cardiology (ESC). Endorsed by: Association for European Paediatric and Congenital Cardiology (AEPC). Eur Heart J. 2015;36(41):2793-867.

22. Forleo GB, Della Rocca DG, Papavasileiou LP, Molfetta AD, Santini L, Romeo F. Left ventricular pacing with a new quadripolartransvenous lead for CRT: earlyresults of a prospective comparison with conventional implant outcomes. Heart Rhythm. 2011;8:31-7.

23. Packer M. Proposal for a new clinical end point to evaluate the efficacy of drugs and devices in the treatment of chronic heart failure. J Card Fail. 2001;7:176-82.

24. Steiner $H$, Geist M, Goldenberg I, Suleiman M, Glikson M, Tenenbaum A, Swissa M, Fisman EZ, Golovchiner G, Strasberg B, Barsheshet A, Israeli Working Group of Pacing and EP. Characteristics and outcomes of diabetic patients with an implantable cardioverter defibrillator in a real world setting: results from the Israeli ICD registry. Cardiovasc Diabetol. 2016;15(1):160

25. Biffi M, Moschini C, Bertini M, Saporito D, Ziacchi M, Diemberger I, Valzania C, Domenichini G, Cervi E, Martignani C, Sangiorgi D, Branzi A, Boriani G. Phrenicstimulation a challenge for cardiac resynchronization therapy. Circ: Arrhythm Electrophysiol. 2009;2(4):402-10.

26. Seifert M, Schau T, Moeller V, Neuss M, Meyhoefer J, Butter C. Influence of pacing configurations, body mass index, and position of coronary sinus lead on frequency of phrenic nerve stimulation and pacing thresholds under cardiac resynchronization therapy. Europace. 2010;12(7):961-7.

27. Hallier MS, Rinaldi CA. First prospective, multi-centre clinical experience with a novel left ventricular quadripolarlead. Europace. 2012;14:365.

28. Champagne J, Healey JS, Krahn AD, Philippon F, Gurevitz O, Swearingen $\mathrm{A}$, Glikson $\mathrm{M}$. The effect of electronic repositioning on left ventricular pacing and phrenic nerve stimulation. Europace. 2011;13(3):409-15.

29. Behar JM, Bostock J, Zhu Li AP, Chin HM, Jubb S, Lent E, Gamble J, Foley PW, Betts TR, Rinaldi CA, Herring N. Cardiac resynchronization therapy delivered via a multipolar left ventricular lead is associated with reduced mortality and elimination of phrenic nerve stimulation: long-term follow-up from a multicenter registry. J Cardiovasc Electrophysiol. 2015;26(5):540-6.

30. Jones MA, Khiani R, Foley P, Webster D, Qureshi N, Wong KC, Rajappan K, Bashir Y, Betts TR. Inter- and intravein differences in cardiac output with cardiac resynchronization pacing using a multipolar LV pacing lead. Pacing Clin Electrophysiol. 2015;38(2):267-74. 
31. Hoppe UC, Casares JM, Eiskjaer H, Hagemann A, Cleland JG, Free-mantle $\mathrm{N}$, Erdmann E. Effect of cardiac resynchronization on the incidence of atrial fibrillation in patients with severe heart failure. Circulation. 2006;114:18-25

32. Lellouche N, De Diego C, Vaseghi M, Buch E, Cesario DA, Mahajan A, Wiener I, Fonarow GC, Boyle NG, Shivkumar K. Cardiac resynchronization therapy response is associated with shorter duration of atrial fibrillation. Pacing Clin Electrophysiol. 2007;30(11):1363-8.

33. Mahajan R, Lau DH, Sanders P. Impact of obesity on cardiac metabolism, fibrosis, and function. Trends Cardiovasc Med. 2015;25(2):119-26.

34. Mahajan R, Lau DH, Brooks AG, Shipp NJ, Manavis J, Wood JP, Finnie JW, Samuel CS, Royce SG, Twomey DJ, Thanigaimani S, Kalman JM, Sanders P. Electrophysiological, electroanatomical, and structural remodeling of the atria as consequences of sustained obesity. J Am Coll Cardiol. 2015;66(1):1-11.

35. Calò L, Martino DeRuvo E, Minati M, Fratini S, Rebecchi M, et al. Acute echocardiographic optimization of multiple stimulation configurations of cardiac resynchronization therapy through quadripolar left ventricular pacing: a tailored approach. Am Heart J. 2014;167:547-54.

36. Szepietowska B, Polonsky B, Sherazi S, Biton Y, Kutyifa V, McNitt S, Aktas M, Moss AJ, Zareba W. Effect of obesity on the effectiveness of cardiac resynchronization to reduce the risk of first and recurrent ventricular tachyarrhythmia events. Cardiovasc Diabetol. 2016;15:93.

37. Cabrera BF, Alzueta RJ, Olaguede RJ, Fernandez-Lozano I, Garcia Guerrero $J$ J, de la Concha JF, et al. Improvement in hemodynamic better programmability with quartet than bi- and tripolar equivalents. Pacing Clin Electrophysiol. 2013;36:963-9.
38. Cai C, Hua W, Ding LG, Wang J, Chen KP, Yang XW, Liu ZM, Zhang S. Association of body mass index with cardiac reverse remodeling and long-term outcome in advanced heart failure patients with cardiac resynchronization therapy. Circ J. 2014;78(12):2899-907.

39. Axelsen LN, Calloe K, Braunstein TH, Riemann M, Hofgaard JP, Liang B, Jensen CF, Olsen KB, Bartels ED, Baandrup U, Jespersen T, Nielsen LB, Holstein-Rathlou NH, Nielsen MS. Diet-induced pre-diabetes slows cardiac conductance and promotes arrhythmogenesis. Cardiovasc Diabetol. 2015;14:87.

40. Sardu C, Santamaria M, Rizzo MR, Barbieri M, di Marino M, Paolisso G, Santulli G, Marfella R. Telemonitoring in heart failure patients treated by cardiac resynchronization therapy with defibrillator (CRT-D): the TELECART Study. Int J ClinPract. 2016;70(7):569-76.

41. Sardu C, Barbieri M, Rizzo MR, Paolisso P, Paolisso G, Marfella R. Cardiac resynchronization therapy outcomes in type 2 diabetic patients: role of MicroRNA changes. J Diabetes Res. 2016:7292564.

42. Sardu C, Marfella R, Santulli G, Paolisso G. Functional role of miRNA in cardiac resynchronization therapy. Pharmacogenomics. 2014;8:1159-68.

43. Umar F, Taylor RJ, Stegemann B, Marshall H, Flannigan S, Lencioni M, De Bono J, Griffith M, Leyva F. Haemodynamic effects of cardiac resynchronization therapy using single-vein, three-pole, multipoint left ventricular pacing in patients with ischaemic cardiomyopathy and a left ventricular free wall scar: the MAESTRO study. Europace. 2016;18(8):1227-34.

44. Shetty AK, Sohal M, Chen Z, Ginks MR, Bostock J, Amraoui S, Ryu K, Rosenberg SP, Niederer SA, Gill J, Carr-White G, Razavi R, Rinaldi CA. A comparison of left ventricular endocardial, multisite, and multipolar epicardial cardiac resynchronization: an acute haemodynamic and electroanatomical study. Europace. 2014;16(6):873-9.

\section{Submit your next manuscript to BioMed Central and we will help you at every step:}

- We accept pre-submission inquiries

- Our selector tool helps you to find the most relevant journal

- We provide round the clock customer support

- Convenient online submission

- Thorough peer review

- Inclusion in PubMed and all major indexing services

- Maximum visibility for your research

Submit your manuscript at www.biomedcentral.com/submit
() Biomed Central 